\title{
Charting the Transient Universe using Radio Continuum Surveys
}

\author{
Jean-Pierre Macquart* \\ ICRAR/Curtin University, Curtin Institute of Radio Astronomy, Perth WA 6845, Australia \\ E-mail: J.Macquartecurtin.edu.au
}

The field of radio transients is exploding with the discovery of diverse new phenomena fuelled by recent advances in telescope and computational capabilities. The desire to maximise time on sky to detect ever more and rarer events drives us to share telescope time with large continuum surveys. We discuss here the advantages of a symbiotic relationship between transients and continuum surveys, and show how an understanding of the time domain can constitute an important facet of continuum survey data with regard to quality control, the interpretation of flux density and spectral information, and the origin of the radio emission. One example at centimetre wavelengths is the presence of intra-day variability, which sifts for the presence of $\sim 1-100 \mu$ as structure and potentially serves as a discriminant of AGN and starburst-related radio emission. We identify and discuss four main issues for the successful integration of transients and continuum surveys.

EXTRA-RADSUR2015 (*)

20-23 October 2015

Bologna, Italy

(*) This conference has been organized with the support of the Ministry of Foreign Affairs and International Cooperation, Directorate General for the Country Promotion (Bilateral Grant Agreement ZA14GR02 - Mapping the Universe on the Pathway to SKA)

${ }^{*}$ Speaker. 


\section{Introduction}

The field of radio transients is presently undergoing a renaissance which owes as much to the increasing prevalence of highly-sensitive wide field-of-view telescopes as it does to commensurate increases in the available computational power needed to exploit their full potential. The importance of this pairing is no better vindicated than by the discovery of Fast Radio Bursts (FRBs; Lorimer et al.2007), whose detection required not only the field of view of the 13-beam receiver on the Parkes radio telescope but the advent of the computational capacity needed to sift for individual bursts from the large data volumes it generated. An enlightening exposition of this coupling as it applies more generally to transients science, presented by Bower (2007), presses the conviction that chance not only favours the prepared mind, but the well-planned and -resourced survey.

An important facet of surveys on next-generation telescopes is the imperative to conduct searches for transients commensally with other surveys. This is driven by the simple desire to spend as much time on sky as possible: the event detection rate for impulsive events is linearly proportional to time on sky, so the more time spent searching, the more transients we find, and the rarer the type of event we are capable of detecting. The desire to utilise the telescope close to $100 \%$ of the time imposes another set of challenges: it requires multiple back-end signal processing paths and a survey strategy that is capable of simultaneously fulfilling all the scientific objectives of the various projects running concurrently on the telescope.

The resourcing and organisation of large-scale surveys is a point of increasing relevance to ongoing widefield projects being undertaken by the Murchison Widefield Array (MWA) and LOFAR (e.g. Wayth et al.2015; Stappers et al.2011), and to those continuum and transients surveys planned on ASKAP, APERTIF and MeerKAT (e.g. TRAPPUM, PIs Stappers \& Kramer; EMU, Norris et al. 2011; CRAFT, Macquart et al. 2010; VAST, Murphy et al. 2013). Full exploitation of the full dates rates of the upcoming SKA1_LOW and SKA1_MID telescopes will present yet more formidable challenges.

Keeping with the spirit of this meeting, the main thrust of this paper is to explore how transients surveys can coexist with other surveys, necessitated by the imperative to share telescope time without compromising either project's scientific objectives. We also explore the various means by which the data products of time-domain studies might complement the scientific objectives of partner surveys. In $\S 2$ we review some recent discoveries in the field of radio transients as means of underlining the importance of various aspects of survey logistics. In section $\S 3$ we consider how transients surveys can coexist with partner surveys, and we present our conclusions in $\S 4$.

\section{Some recent discoveries}

The field of radio transients encompasses a diverse range of objects and timescales. Here we draw the reader's attention to the vibrancy of the field by cherry-picking three recent developments across this broad discipline. More exhaustive summaries of the prospects for transients science in the coming $\sim 5-10$ years are contained in Fender et al. (2015) and references therein.

We also describe here some examples of variable and transient sources likely to be prevalent in continuum surveys, and whose effects will be manifest and will likely need to be accounted for. 


\subsection{Fast Radio Bursts}

Fast Radio Bursts (FRBs; Lorimer et al. 2007; Thornton et al. 2013) are a newly-discovered class of impulsive events with durations of order milliseconds whose dispersion measures place them prima facie at cosmological distances. Several other properties of these events, including their scattering characteristics and source counts present compelling evidence in support of their origin at cosmological distances (Macquart \& Koay 2013; Luan \& Goldreich 2014; Macquart \& Johnston 2015).

There is impressive scope to use FRBs as high-precision probes of cosmology to address contemporary issues such as the "missing baryon" problem (see McQuinn 2013; Macquart et al. 2015), and possibly even to place constraints on the evolution of $\Omega_{\Lambda}$ (Zhuo et al. 2014). There exists the possibility to map the distribution of baryons in the Local Universe in the same manner that pulsars have been used to map the ionized interstellar medium of our own Galaxy. Such ambitious scientific objectives can only be realised with large numbers, of order thousands, of events. It appears likely that the requisite detection rate will only be realised with SKA1 coupled with a high-time resolution backend capable of tiling a significant fraction of the telescope primary beam.

\subsection{Radio-emitting cataclysmic variables}

A recent JVLA survey has detected transient radio emission from dwarf novae. The survey detected outbursts in three out of four systems examined, with flux densities in the range $15-$ $150 \mu \mathrm{Jy} \mathrm{beam}^{-1}$ for objects ranging in distance between 100 and $330 \mathrm{pc}$ (Coppejans et al. 2015). It is likely that the systems are undetectable in quiescence, so will only be detectable as transients.

Dwarf novae are numerous within our Galaxy, and serve as nearby laboratories to study accretion in a non-relativistic regime. They thus offer an important link in understanding the fundamental physics of accretion and ejection; comparison with neutron star and black hole systems can be used to examine how jet launching is affected by the depth of the gravitational potential well.

\subsection{Ionospheric ducts and large-scale structures}

A serendipitous discovery that emanated from studies of widefield data taken on a cadence of $\sim 10$ s from the MWA was the detection of large-scale organised structures in the upper terrestrial ionosphere (Loi et al.2015). The existence of these structures was inferred from the large-scale organised motions in the positions of sources due to image wander caused by ionospheric scintillation. This revealed that, far from being random, the structures responsible for these motions are coherent on scales of order $5^{\circ}$, and consistent with the topology and orientation of the geomagnetic field at an altitude of $\sim 600 \mathrm{~km}$ (Loi et al. 2015).

This discovery demonstrates the propensity of widefield high-time resolution data to make discoveries in entirely unanticipated domains. It is a point particularly germane to the topic of this meeting, namely the utility of future continuum surveys, since this discovery was made from the data obtained for the GaLactic and Extragalactic MWA (GLEAM) continuum survey (Wayth et al. 2015).

\section{4 “Contaminants" in continuum surveys}

Radio sources may also exhibit variability due to propagation of their radiation through the turbulent plasmas that pervade both the solar system and interstellar space. Such variability, if 
detectable by the survey, can be used to supplement imaging data by providing information on angular scales that are usually far smaller than those resolvable with conventional interferometers.

At frequencies below $\approx 300 \mathrm{MHz}$, sources smaller than $\sim 0.1-1^{\prime \prime}$ are subject to the effects of interplanetary scintillation due to turbulence in the solar wind. The effect gives rise to variations on timescales $\sim 0.1-2 \mathrm{~s}$ and is easily observable for objects within $\sim 40^{\circ}$ of the Sun. The effect is strongly frequency-dependent; as the frequency decreases the intensity scintillations decrease in timescale, increase in angular resolution and are more prevalent at greater distances from the Sun.

The phenomenon of interstellar scintillation, on the other hand, is observed at frequencies up to $\approx 15 \mathrm{GHz}$, and typically gives rise to flux density variations on timescales of hours to days (to weeks at frequencies below $\approx 2 \mathrm{GHz}$ ) for objects located off the Galactic plane. Interstellar scintillation is relevant to objects with source sizes $\sim 100 \mu \mathrm{as}(v / 5 \mathrm{GHz})^{-2}$ or less.

Scintillation bears a number of consequences for continuum surveys, and its effects can present both a help and a hindrance:

- Scintillation variability acts as a cheap form of widefield VLBI. Any sufficiently compact objects will be subject to the effects of interstellar scintillation, and its detectable presence heralds the existence of ultra-compact substructure within the emission region. As interstellar scintillation typically probes substructure on angular scales $0.1-100 \mu$ as, it offers information on the source typically two to three orders of magnitude smaller than accessible using conventional VLBI. As such, scintillation is potentially an excellent and cheap discriminator between AGN and starburst-fuelled radio emission.

- The timescale of interstellar scintillation is typically large relative to the interferometer imaging timescale, so the flux densities of objects affected by this process can differ significantly from their intrinsic values. At frequencies $\sim 5 \mathrm{GHz}$ the scintillation can modulate the source flux density by amounts exceeding $100 \%$ of the mean value of the flux density present in the compact component. This point is discussed further below in $\$ 2.4 .2$.

- The spectral indices of sources affected by interstellar scintillation will be in error, thus hindering source classification schemes that rely heavily on spectral information. Measurements of the source flux density at multiple epochs are necessary to circumvent this problem.

- The effects of interplanetary scintillation (IPS) for metre-wavelength interferometers may be mitigated by integrating on timescales substantially longer than seconds or by observing at large solar elongations (but see the caveat below). However, telescopes capable of imaging with sub-second cadence can exploit the effects of interplanetary scintillation as a means of accessing sub-arcsecond structure, as demonstrated to great effect by Morgan (these proceedings).

\subsubsection{Night-time IPS}

It is generally believed that the effects of IPS are ignorable at frequencies $v \gtrsim 100 \mathrm{MHz}$ for observations at solar elongations exceeding $\approx 50^{\circ}$. However, Kaplan et al. (2015) report the detection of IPS in night-time observations of sources at solar elongations of $90^{\circ}-100^{\circ}$ at $155 \mathrm{MHz}$. Furthermore, the scintillations were intermittent in the sense that the variations occurred only for a 
duration of approximately 30 minutes, with the sources exhibiting root-mean-square modulations of about $25 \%$ of their mean flux densities. Kaplan et al. (2015) argue that the phenomenon likely represents the passage of a coronal mass ejection across the foreground of the observed field.

It is apposite that the observations in which this discovery was made were being undertaking specifically for studies of the Epoch of Reionization, and that the observations were made at a time when the effects of IPS were believed to be negligible. This discovery serves as a reminder that the effects of scintillation are often intermittent and, if not properly accounted for, may pose a deleterious effect on applications requiring high degrees of precision, such as Epoch of Reionization observations.

\subsubsection{Quasar intra-day variability}

The phenomenon of quasar intra-day variability (IDV) is a manifestation of interstellar scintillation. The effect is relevant to AGN with compact sources: the MASIV VLA survey of several hundred steep- and inverted-spectrum AGN in the range $\approx 20-1000 \mathrm{mJy}$ found that over $56 \%$ of all sources exhibited IDV in at least one of four epochs over the course of a year (Lovell et al. 2003, 2008). Most of the AGN detected in MASIV exhibited variability on timescales of order days with amplitudes of order $1 \%$ at $5 \mathrm{GHz}$. This amplitude is considerably lower than the $\sim 100 \%$ modulations theoretically possible because the compact (scintillating) component of the source comprises only a small fraction of the total flux density observed (on the arc-second scales that the VLA is sensitive to).

Intermittency is also a common feature of intra-day variability. MASIV found that individual sources tend to exhibit IDV intermittently (Lovell et al. 2008), with only $20 \%$ of the sample exhibiting IDV at any one time. The properties of the turbulence depend on the line of sight: although most sources exhibited scintillation on timescales of order a day, some six sources exhibited variations on timescales of order only a few hours (Koay et al. 2012). Finally, as an extreme demonstration of the intermittency of interstellar scintillation, we note that the most extreme intra-hour variable quasar known, J1819+3845 (Dennett-Thorpe \& de Bruyn 2000), which was observed to exhibit modulations of up to $40 \%$ on timescales down to 20 minutes over the course of nearly a decade, has now effectively ceased to scintillate. It now exhibits rms modulations only $\approx 1 \%$ of its mean flux density (de Bruyn \& Macquart 2015).

\subsection{Spectral line variability}

Finally, although not specifically the focus of this meeting, we draw attention to the intriguing possibility that HI and other spectral-line surveys may detect variability in the foreground absorption against bright radio continuum sources. Such variations have been reported by Wolfe, Davis \& Briggs (1982) and Kanekar \& Chengalur (2001) but the phenomenon appears to have largely been ignored by the community. However, the detection of such variability in other systems opens up the possibility of probing the small-scale structure of the absorbing region on scales down to milli-arcseconds (e.g. Macquart 2005).

\section{Four essential elements of transients surveys}

As we progress towards the SKA era there is considerable discussion centred around areas of 
commensality between large surveys, driven by the fact that the total number of available observational hours is dwarfed by the time required by Key Science Projects to fulfill their scientific objectives.

The purpose of this section is to advance some practical suggestions to ensure that the symbiosis between continuum and transients surveys yields maximum benefit to both. We distill this down to four essential propositions.

\subsection{Time on sky}

Should future transients surveys be dedicated and planned around a fixed schedule, or should they be opportunistic, seeking to detect events no matter what primary observing program happens to be running on the telescope at the time? In the field of radio transients, there is no substitute for time on sky. It has been argued elsewhere (e.g. Fender et al.2015) that it is reasonable to expect that a dedicated survey for transients might utilise at most about $10 \%$ of the total available telescope time. However, a transients program that is capable of sharing resources and is able to access data from all telescope operations would therefore expect to detect roughly ten times as many transients or, conversely, detect events that are ten times rarer. The imperative to build continuum surveys (and transients pipelines) capable of extracting transient events is clear.

An important consideration relating to survey strategy is driven by the need to detect the flux density difference necessary to identify an object as a transient. Often the question arises whether a particular survey should be sequential or piecemeal: is it preferable to survey the sky sequentially, field-by-field or should it tile the survey region in multiple passes until the requisite sensitivity is reached? We argue that a piecemeal approach is beneficial to continuum survey science to identify those sources that are variable (see, e.g., §2.4.2). For projects searching for events that occur on a timescale slow compared to the time required to image a field, it is highly desirable that the survey which is being piggybacked on either adopts a strategy that revisits that same sky on multiple occasions or that there is a reference image of the field. On the other hand, this is not a consideration for pipelines searching for transients on timescales less than the correlator integration time ("fast transients"), since each imaging observation will comprise a large number of independent "realisations" of the variable sky, and it is easy to detect events whose timescale is short compared to the total data span.

\subsection{Near real-time response and localisation}

A central question is to what extent the pre-existing primary survey being conducted on the telescope should need to accede to overrides in favour of dedicated observations of transient events. The traditional method of determining whether an override should occur at existing observatories (e.g. the JVLA, VLBA and ATCA) is based on a comparison of the Time Allocation Committee ratings of the pre-existing and overriding proposals. However, we suggest that, in the era of survey science, it would be more useful to examine both the importance and the urgency of the interruption, thus recognising the time-critical nature of the interruption. Often the program being interrupted is not time-critical: radio continuum surveys are usually concerned with phenomena that evolve on timescales comparable to the age of the Universe, not on timescales of hours or less.

A major driver of transient followup relates to the localisation of events. Localisation is everything in this game; it enables followup of an event across the entire electromagnetic spectrum, 
especially at optical wavelengths. For many types of transient events the maximum scientific impact only occurs when it is possible to compare the evolution of an event's emission across a large fraction of the spectrum.

When planning a survey to operate commensally on the telescope there are a number of considerations that relate to time-variable phenomena:

1. How much flexibility should be built into a survey to allow opportunistic interruptions to its schedule?

2. When responding to a trigger, do the observations need the entire sensitivity of the telescope, or can the objectives be satisfied using a fraction of baselines? In many instances transients are initially bright and sensitivity is less of an issue than good calibration.

3. What kind of trigger is the telescope responding to: is its primary purpose to measure the evolution of an outburst, or is it to obtain a precise localisation for an event that was detected by another facility? The nature of the trigger will determine the type of telescope resources required in the response: event localisation, for example, may only require only the outlying elements of the array. VLBI will be an essential followup component for some types of events.

4. How should a transients detection pipeline assign priority to triggers, and what is the threshold for a pre-existing survey to act upon it?

5. Should there be a pre-existing agreement between survey groups sharing telescope resources on the algorithm that decides how real-time triggers should be handled? (Or should this be brokered by the observatory?)

These points all stress the need for communication amongst survey teams in the planning stage.

\subsection{Characterisation}

Event characterisation is closely coupled with the subject of the previous subsection, namely localisation and the response to event triggers. However, it extends to other burst properties including: the spectrum, polarization properties, and the possible existence of finer timescale structure. Is the burst coincident with position of known transient sources, such as CV stars, flare stars, X-ray binaries or magnetars?

An essential component of event characterisation is a memory buffer that allows us to retroactively reconstruct images or high-time resolution data in response to a trigger. A transients buffer is effectively a "time machine" that enables us to respond to transient alerts with some degree of latency. This is essential when responding to particular types of events in which there may be considerable delay (i.e.tens of seconds) between event reception and formal detection, such as gravitational wave events. Transients buffers will be inherent components of both SKA1_MID and SKA1_LOW. 


\subsubsection{Symbiosis}

The frequent necessity to link the emission of a radio transient to as much of the rest of the electromagnetic spectrum as possible bears a number of advantages for other survey programs running on the same telescope. It brokers pre-existing arrangements between the users of the telescope and a number of multi-wavelength facilities. It also ensures that fields studied in detail for a transient emission will contain a suite of multi-wavelength data available for studies of other sources within the field.

In the SKA era the transients community envisages brokering followup agreements with a number of large facilities including

- The Large Synoptic Survey Telescope (LSST)

- Extremely Large Telescopes (e.g. the Giant Magellan Telescope, OWL)

- The James Webb Space Telescope (JWST)

- The Atacama Large Millimeter Array (ALMA)

- X-ray \& gamma-ray facilties

- Advanced LIGO

This will be supplemented by dedicated followup facilities, such as MeerLicht, a $0.65 \mathrm{~m} 2$ sq.deg. field of view optical telescope that is intended to provide simultaneous optical-radio observations of the night sky in conjunction with MeerKAT (see http://www.ast.uct.ac.za/meerlicht/MeerLICHT.html).

Finally, we point out the current census of opinion within the SKA transients community is one in which all transients events are shared with the entire community, so that continuum surveys will be able to make use of identifications for their own scientific purposes. This is a model broadly embraced in the wider transients community (e.g. by SWIFT and LSST).

\subsection{Followup - milking the science}

A final but obvious facet of an effective transients survey strategy is the provision of an event archive with an effective user interface. It is an open question how such data should be combined with the data products of other surveys, if at all.

\section{Concluding remarks}

Most transients science lends itself to being conducted commensally, with few notable exceptions (e.g. the examination of specific targets, or perhaps the Galactic Centre), so it is vital that we learn how to mesh continuum and transients surveys together.

There is a potential for a highly productive symbiosis between survey communities because variability information can often provide additional detail about the characteristics of particular sources appearing in the survey data. We suspect that the occurrence of interstellar scintillation as a probe of micro-arcsecond scale structure of AGN will be important instance of this. Moreover, the analysis of data at high time resolution can often identify defects, artefacts and phenomena 
in the data that might otherwise have gone unnoticed and may affect data quality; an example presented in this article is the serendipitous discovery of intermittent inter-planetary scintillation at elongations exceeding $90^{\circ}$ from the Sun.

Usually the simple detection of a transient event is, by itself, uninteresting, and followup observations are often necessary to characterise the event in more detail. This requirement poses a number of questions about how commensally-run surveys should interact with each other in responding to triggers, especially if the trigger response is to be automated. This issue is more social and operational than it is technical, and it is best addressed in the survey planning stage with the help of good communication between the various survey teams. Early teething experiences with SKA pathfinders and precursors will be vital in shaking down this aspect of SKA operations.

Acknowledgments The author thanks James Miller-Jones for stimulating discussions and comments on the manuscript.

\section{References}

Bower, G.C. 2007, Science, 318, 759

de Bruyn, A.G. \& Macquart, J.-P. 2015, A\&A, 574, 125

Coppejans et al. 2015, MNRAS, 451, 3801

Dennett-Thorpe, J. \& de Bruyn, A.G. 2001, ApJ Lett, 529, L65

Fender, R. et al. 2015, PoS(AASKA14)051

Kaplan, D.L. et al. 2015, ApJ Lett, 809, L12

Kanekar, N. \& Chengalur, J. 2001, MNRAS, 325, 631

Loi, S.T. et al. 2015, Geophys. Res. Lett., 42, 3707

Lorimer, D.R., Bailes, M., McLaughlin, M.A., Narkevic, D.J. \& Crawford, F. 2007, Science, 318, 777

Lovell, J.E.J. et al. 2003, AJ, 126, 1699

Lovell, J.E.J. et al. 2008, ApJ, 689, 108

Luan, J. \& Goldreich, P. 2014, ApJ, 785, L26

Macquart, J.-P. 2005, A\&A, 433, 827

Macquart, J.-P. et al. 2010, PASA, 27, 272

Macquart, J.-P. et al. 2015, PoS(AASKA14)055

Macquart, J.-P., \& Johnston, S. 2015, MNRAS, 451, 3278

Macquart, J.-P. \& Koay, J.Y. 2013, ApJ, 776, 125 
McQuinn, M. 2014, ApJ, 780, L33

Murphy, T. et al. 2013, PASA, 30, 6

Norris, R.P. et al. 2011, PASA, 28, 215

Stappers, B.W. et al. 2011, A\&A, 530, A80

Thornton, D., Stappers, B., Bailes, et al. 2013, Science, 341, 53

Wayth, R.B. et al. 2015, PASA, 32, 25

Wolfe, A.M., Davis, M.M. \& Briggs, F.H. 1982, ApJ, 259, 495

Zhou, B., Li, X., Wang, T., Fan, Y.-Z. \& Wei, D.-M. 2014, Phys. Rev. D., 89(10), 107303 